Article

\title{
Quantitative Structure-Activity Relationships Predicting the Antioxidant Potency of 17 $\beta$-Estradiol-Related Polycyclic Phenols to Inhibit Lipid Peroxidation
}

\author{
Laszlo Prokai $^{1, *}$, Nilka M. Rivera-Portalatin ${ }^{1, \dagger}$ and Katalin Prokai-Tatrai ${ }^{2}$
}

1 Department of Molecular Biology and Immunology, University of North Texas Health Science Center, Fort Worth, TX 76107, USA; E-Mail: nilka.rivera1@upr.edu

2 Department of Pharmaceutical Sciences, UNT System College of Pharmacy, University of North Texas Health Science Center, Fort Worth, TX 76107, USA; E-Mail: katalin.prokai@unthsc.edu

$\dagger$ Current address: Department of Chemistry, University of Puerto Rico, Mayagüez, PR 00681, Puerto Rico.

* Author to whom correspondence should be addressed; E-Mail: laszlo.prokai@unthsc.edu; Tel.: +1-817-735-2206; Fax: +1-817-735-2118.

Received: 13 December 2012; in revised form: 3 January 2013 / Accepted: 5 January 2013 / Published: 11 January 2013

\begin{abstract}
The antioxidant potency of $17 \beta$-estradiol and related polycyclic phenols has been well established. This property is an important component of the complex events by which these types of agents are capable to protect neurons against the detrimental consequences of oxidative stress. In order to relate their molecular structure and properties with their capacity to inhibit lipid peroxidation, a marker of oxidative stress, quantitative structure-activity relationship (QSAR) studies were conducted. The inhibition of $\mathrm{Fe}^{3+}$-induced lipid peroxidation in rat brain homogenate, measured through an assay detecting thiobarbituric acid reactive substances for about seventy compounds were correlated with various molecular descriptors. We found that lipophilicity (modeled by the logarithm of the $n$-octanol/water partition coefficient, $\log \mathrm{P}$ ) was the property that influenced most profoundly the potency of these compounds to inhibit lipid peroxidation in the biological medium studied. Additionally, the important contribution of the bond dissociation enthalpy of the phenolic $\mathrm{O}-\mathrm{H}$ group, a shape index, the solvent-accessible surface area and the energy required to remove an electron from the highest occupied molecular orbital were also confirmed. Several QSAR equations were validated as potentially useful exploratory tools for identifying or designing novel phenolic antioxidants
\end{abstract}


incorporating the structural backbone of $17 \beta$-estradiol to assist therapy development against oxidative stress-associated neurodegeneration.

Keywords: $17 \beta$-estradiol; estrogens; lipid peroxidation; phenolic antioxidant; oxidative stress; QSAR; TBARS

\section{Introduction}

Oxidative stress (OS) has been implicated as a key factor to the initiation and/or progression of numerous pathological processes and premature aging [1]. OS-initiated lipid peroxidation (LPO) has been extensively studied as a potential biomarker for this detrimental process, since lipids are particularly sensitive to OS $[2,3]$. The high unsaturated lipid content of the brain, coupled with high oxygen utilization, elevated redox-active metal ions concentration and relatively poor antioxidant defense, makes this organ quite vulnerable to this process and the associated pathologies [4]. One of the most promising approaches to combat OS and, thus, prevent the resultant LPO is the use of antioxidants that can either "catch" free radicals or chelate excessive redox metals that can provoke OS via the formation of reactive oxygen species (ROS) [5].

Estrogens are unique among steroids in terms of having the phenolic A-ring that allows for eliciting antioxidant activity similar to that of simple phenolic antioxidants, such as vitamin $\mathrm{E}$ or butylated hydroxytoluene [6,7]. It has become evident that the ability of $17 \beta$-estradiol $(E 2,1)$, the main human estrogen (Figure 1), to act as a direct free radical scavenging antioxidant is an important element in the cascade of neuroprotective events by which E2 provides protection against OS both in vitro [7-9] and in vivo [10-12], although the latter is not without controversy [13]. Nevertheless, it has been shown that E2 and related compounds are able to directly scavenge free radicals and, thereby, alleviate OS independently of nuclear estrogen receptor (ER) activation $[14,15]$. Only the free phenolic $\mathrm{OH}$ of the A-ring is the prerequisite for this activity. This seminal finding was first recognized by Behl et al. [16]. An important new antioxidant cycle for estrogens to shield against free radicals was recently discovered showing that estrogens can actually be regenerated after sweeping up free radicals [10,17]. Additional proposed mechanisms include the synergistic interaction between estrogens and glutathione [18], as well as chelating redox metals, particularly by estrogens having an unsaturated B-ring [19]. It should also be noted that the indirect antioxidant actions of these biomolecules have also been confirmed; most prominently via the upregulation of the endogenous antioxidant defense system [20].

There are several caveats associated with the use of E2 for antioxidant therapy targeting the brain; for example, E2 lacks adequate oral bioavailability [21] and its administration also produces unwanted hormonal and other potentially detrimental side-effects in the periphery [22,23]. In the search for new E2-derived agents that preserve E2's strong antioxidant property [24] without peripheral liability, studies with limited scopes have been conducted with E2 derivatives exhibiting diminished or no ER binding affinity [25]. It has been known that manipulation of ER-binding can be done by introducing substituents to and/or performing isomerization on the E2 structure [26,27]. In particular, the fundamental work of Miller et al. concluded that the ER-binding affinity (i.e., estrogenic activity) and 
antioxidant efficacy can be largely separated with the presence of bulky substituents on the phenolic A-ring of E2 [27]. Quantitative structure-activity relationship (QSAR) studies connecting the estrogen structure and ER-binding ability necessary to trigger the well-documented genomic actions of estrogens have been reported, most particularly to identify estrogenic endocrine disruptors [28-30].

In vitro studies to establish the structural requirements for antioxidant potency have used small libraries of compounds [15,31,32]. In order to facilitate drug discovery-driven efforts to search and identify potentially useful antioxidants using E2 and estrone $(\mathrm{E} 1,2)$ as templates (Figure 1), we aimed at performing QSAR studies using an extended library of E2 and E1 derivatives or analogues (3-60), as well as related polycyclic phenols (61-70). The QSAR modeling was based on the construction of predictive models for the inhibition of $\mathrm{Fe}^{3+}$-induced LPO in rat brain homogenate by these compounds [33], assessed through the detection of thiobarbituric acid reactive substances (TBARS), as an experimental measure for antioxidant potency. The TBARS assay is a widely adopted and sensitive method for measuring the extent of LPO [34]. The oxidation of unsaturated fatty acids leads to the formation of malondialdehyde (MDA) as a breakdown product [35]. The reaction of MDA with thiobarbituric acid (TBA) produces a pink chromogen when heated at low $\mathrm{pH}$ with a typical maximum absorbance at $532 \mathrm{~nm}$ [34]. The MDA-TBA complex measured by the TBARS assay is considered a gauge for lipid peroxide $(\mathrm{LOOH})$ formation [36]. Based on this established experimental model, the goal of the present work was to find QSARs that could approximate through a theoretical approach whether a particular estrogen derivative or related phenolic compound would be a more or less potent antioxidant against LPO than E2 (1). These models then may serve as exploratory tools for identifying and designing novel phenolic antioxidants via utilizing the structural backbone of the parent steroid.

Figure 1. Chemical structure of the 17ß-estradiol (E2) and estrone (E1).
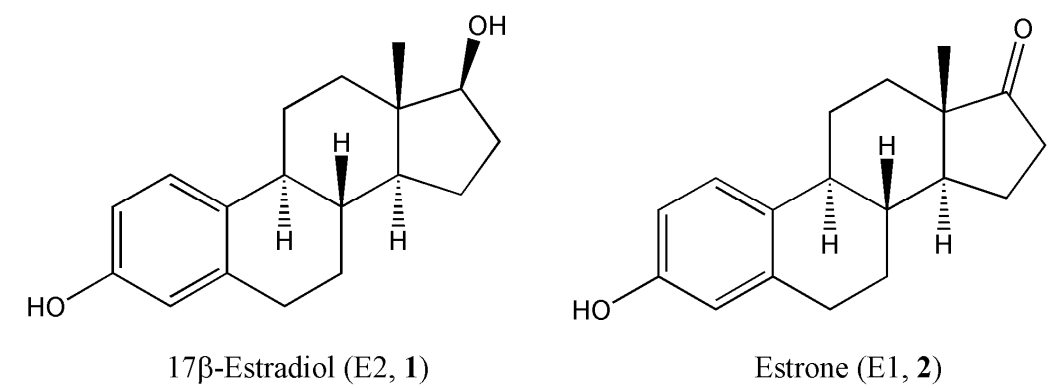

\section{Results and Discussion}

\subsection{Construction of QSAR Models}

The experimental antioxidant potencies, expressed as $\mathrm{IC}_{50}$ values for the inhibition of LPO measured via the TBARS assay in ovariectomized (OVX) rat brain homogenate against $\mathrm{Fe}^{3+}$-induced LPO [25,37,38], of the selected compounds (1-70) are given in the Supplementary Information (Table S1, which is a spreadsheet in Microsoft Excel format also displaying the chemical structures). Specifically, $\mathrm{IC}_{50}$ of a compound represents the concentration that inhibits $50 \%$ of LPO; thus, a smaller number represents a higher potency in this regard. In comparison with alternative chemometric and cheminformatics tools, the advantage of a descriptor-based approach for the development of predictive 
QSAR models focusing on LPO inhibitory activity has been shown recently [39]. Therefore, we applied the latter strategy to pursue our computational study reported here. The negative logarithm of the $\mathrm{IC}_{50}$ value (in molar concentration, $\mathrm{M}$ ) was chosen as the dependent variable, and various descriptors of the test compounds available through the Project Leader module of the CAChe software were considered as independent variables for the creation of QSAR models:

$$
\begin{gathered}
-\log \left(\mathrm{IC}_{50}-\mathrm{TBARS}\right)=13.6( \pm 3.4)-0.179( \pm 0.042) \cdot \mathrm{BDE}(r=0.46, F=18.11, p<0.001) \\
-\log \left(\mathrm{IC}_{50}-\mathrm{TBARS}\right)=-2.69( \pm 0.36)+0.118( \pm 0.021) \cdot \mathrm{SI}_{\kappa 1}(r=0.55, F=29.85) \\
-\log \left(\mathrm{IC}_{50}-\mathrm{TBARS}\right)=-3.03( \pm 0.41)+0.00728( \pm 0.0031) \cdot \mathrm{SA}(r=0.56, F=30.97) \\
-\log \left(\mathrm{IC}_{50}-\mathrm{TBARS}\right)=-2.27( \pm 0.27)+0.295( \pm 0.052) \cdot \log \mathrm{P}(r=0.57, F=32.42) \\
-\log \left(\mathrm{IC}_{50}-\mathrm{TBARS}\right)=6.48( \pm 3.42)+0.237( \pm 0.0547) \cdot \log \mathrm{P}-0.105( \pm 0.041) \cdot \mathrm{BDE} \\
(r=0.58, F=20.83) \\
-\log \left(\mathrm{IC}_{50}-\mathrm{TBARS}\right)=6.23( \pm 2.63)+0.288( \pm 0.048) \cdot \log \mathrm{P}+0.988( \pm 0.304) \cdot \mathrm{HOMO} \\
(r=0.64, F=23.78) \\
-\log \left(\mathrm{IC}_{50}-\mathrm{TBARS}\right)=2.56( \pm 0.36)+0.186( \pm 0.102) \cdot \log \mathrm{P}+0.0522( \pm 0.0421) \cdot \mathrm{SI}_{\kappa 1} \\
(r=0.58, F=17.10) \\
-\log \left(\mathrm{IC}_{50}-\mathrm{TBARS}\right)=18.5( \pm 4.3)+0.209( \pm 0.050) \log -0.130( \pm 0.037) \cdot \mathrm{BDE}+ \\
1.16( \pm 0.29) \cdot \mathrm{HOMO}(r=0.71, F=22.60)
\end{gathered}
$$

The best statistical models are shown in Equations 1-8. We believe that the somewhat modest correlations were due to the combination of limited structural diversity in the training set and confines of the in vitro experimental procedure relying on an actual, heterogeneous biological medium [33] rather than a well-defined chemical model for LPO [40]. Nevertheless, all of them satisfied the requirement for statistical significance with $p<0.001$ from analysis of variance (ANOVA). The values of phenolic O-H's bond dissociation enthalpy (BDE, kcal/mol), a shape index ( $\kappa$-type, first order, $\mathrm{SI}_{\kappa 1}$ ), the solvent-accessible surface area $\left(\mathrm{SA}, \AA^{2}\right.$ ), lipophilicity (expressed as the logarithm of the $n$-octanol/water partition coefficient; $\log \mathrm{P})$, and the eigenvalues of a frontier orbital $(\mathrm{HOMO}, \mathrm{eV})$ were the descriptors present in the QSAR models obtained, and these descriptors were also included in Table S1.

The first four equations represent models created from the use of only a single molecular descriptor. Equation 4 provided the largest $F$-value (the ratio of the model's explained variance to its unexplained variance, considering $F$ of 15 as threshold value for model selection). This indicated that $\log \mathrm{P}$ (i.e., a descriptor related to lipophilicity) had the best predictive value among parameters found to give the best one-descriptor Equations (1-4; $\mathrm{BDE}, \mathrm{SI}_{\kappa}$, $\mathrm{SA}$ and $\log \mathrm{P}$ ), confirming thereby the previously established significance of lipophilicity regarding LPO inhibition [40]. In addition, $\log$ P was a steady descriptor included, when equations of acceptable statistical significance were searched using two or more independent variables, while other variables in these QSAR models were either BDE (Equation 5), HOMO (Equation 6), $\mathrm{SI}_{\kappa 1}$ (Equation 7), or BDE and HOMO (Equation 8), respectively. 
Altogether, inclusion of descriptors other than $\log \mathrm{P}$ in Equations 5-8 decreased the F-values but, with the exception of including $\mathrm{SI}_{\kappa 1}$ (Equation 7), improved correlation (i.e., increased the $\mathrm{r}$ value).

The extended spin distribution in the phenoxyl radical ( $\left.\mathrm{ArO}^{\circ}\right)$ derived from the parent phenolic antioxidant (ArOH; e.g., E2) after it donates its $\mathrm{H}$ from the phenolic $\mathrm{OH}$ to a free radical to terminate the propagation of a radical reaction has been suggested to be an important contributor for the radical scavenging activity [41,42]. A smaller value of this parameter projects a more stable $\mathrm{ArO}^{\circ}$ and, consequently, better antioxidant potency [40]. Nevertheless, correlation of BDE with extended spin distribution, as well as with the enthalpy of single-electron transfer and the ionization potential (IP) were also noted [40]. Therefore, the BDE was considered for the construction of QSAR models in this context. The BDE increases with the increasing electron withdrawal by the substituents surrounding $\mathrm{ArOH}$; in other words, $\mathrm{O}-\mathrm{H}$ bond is weakened by increasing the electron density and strengthened by decreasing the electron density within the bond [43]. Accordingly, electron donating group(s) on the A-ring of an estrogen should positively impact the antioxidant potency compared to that of the unsubstituted E2 (1). Concurring, Equations 1, 5 and 8 correctly predict that the inhibition of LPO decreases with increasing BDE. This tendency is expected, because compounds that can easily donate the hydrogen of the phenolic $\mathrm{OH}$ to break the cascade of radical-mediated reactions are those with low BDE. This process is schematically shown in Figure 2 [44]; where LH represents a lipid molecule, $\mathrm{LOO}^{\circ}$ is the product of a very fast $\mathrm{O}_{2}$-addition to the chain initiator [45] formed by the reaction $\mathrm{LH} \rightarrow \mathrm{L}^{\bullet}$ upon the attack by $\mathrm{ROS}$, and $\mathrm{LOOH}$ is lipid hydroperoxide. The chain-breaking reaction $\mathrm{ArOH}+\mathrm{LOO}^{\circ} \rightarrow \mathrm{ArO}^{\circ}+\mathrm{LOOH}$ prevents $\mathrm{LPO}$ cycle propagated by an $\mathrm{H}$-atom exchange reaction $\left(\mathrm{LOO}^{*}+\mathrm{LH} \rightarrow \mathrm{LOOH}+\mathrm{L}^{*}\right)$ that regenerates the chain initiator. The pathway drawn in red represent the actual chain-breaking $\mathrm{H}$-atom transfer, while the blue portion of Figure 2 implicates the conversion of the phenoxyl radical ( $\left.\mathrm{ArO}^{\circ}\right)$ back to the phenolic compound $(\mathrm{ArOH})$ by an endogenous reductant $\mathrm{AH}$ such as ascorbate, which is converted to its oxidized form $\mathrm{A}^{\prime}$ in the process [44].

Figure 2. Schematic illustration of lipid peroxidation (LPO) inhibition by phenolic antioxidants.

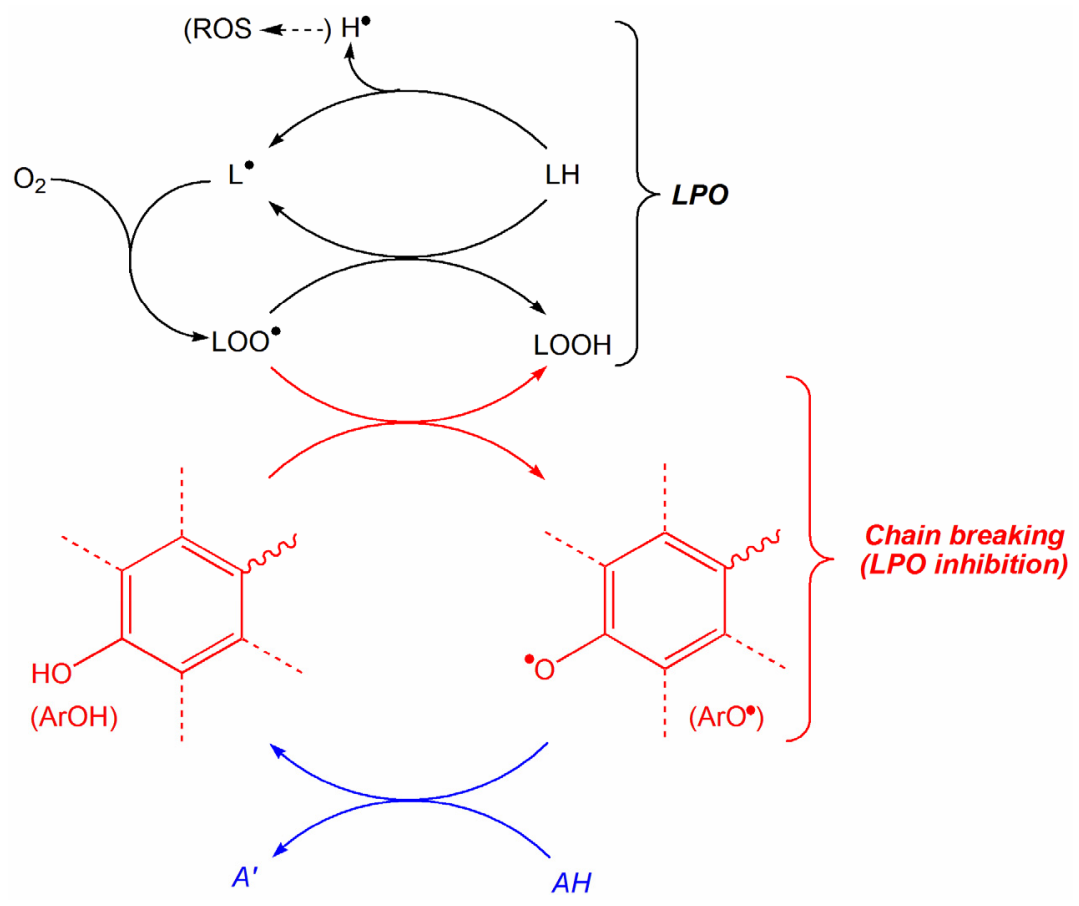


The HOMO energy (the energy required to remove an electron from the highest occupied molecular orbital) has also been connected to the ability of a phenolic compound to donate electrons to free radicals. According to Koopman's theorem and the molecular orbital theory [40], it determines the IP. Therefore, the involvement of the HOMO energy as descriptor refining two different QSAR models (Equations 6 and 8, respectively) was not unexpected, although it did not qualify alone to be among the one-parameter equations giving statistically acceptable correlation. Nevertheless, it was noticeable that correlation increased when this descriptor was also used in the two-parameter equations in addition to $\log \mathrm{P}$ and BDE, respectively. It is noteworthy that the best three-parameter equation also included HOMO (Equation 8) and, provided a larger r-value than BDE and $\log \mathrm{P}$ without this descriptor (Equation 5). The influence of a topological index related to the shape of a molecule was also revealed. The $\mathrm{SI}_{\kappa 1}$ quantifies the number of cycles in the compound [46,47]. Equations 2 and 7 predict that a large $\mathrm{SI}_{\kappa 1}$ value improves the antioxidant potency of an E2-related polycyclic phenol. SA also gave a good correlation and had apparent descriptive value considering the TBARS assay as a measure of LPO inhibition by these compounds. These descriptors were calculated at an optimized geometry in water using the conductor-like screening model (COSMO) for solvation [48]. Equation 3 suggests that a higher antioxidant activity could be obtained with molecules having a higher SA.

\subsection{Validation of QSAR Models (Equations 1-8)}

An important step in the QSAR modeling is to validate the obtained models. An initial validation was carried out for the best models (Equations 1-8) through randomly leaving out $10 \%$ of compounds from the training set $[49,50]$ (data not shown). The equations fitted to this reduced training set with the same descriptors yielded slightly different regression coefficients, but the correlation coefficients of the equations remained similar to those obtained with all entities (1-70) included. In addition, the lack of chance correlations in the reported QSAR models was also ensured by analyzing the equations after randomization of the experimental values.

In a more rigorous validation that was independent from our training set, the obtained QSAR equations were used to predict the relative antioxidant potencies of A-ring substituted estrogens and compared with those obtained by Badeau et al. [32]. These authors analyzed a group of estrogens for their antioxidant capacity and reported the potencies of the compounds as more active or less active than E2 (1). The $\mathrm{IC}_{50}$ 's for the TBARS of these compounds were predicted using QSAR Equations 1-8 and compared with the results reported. The validity of the predictions was determined based on the number of false positives (compounds that were less potent than E2 but were predicted to be more potent), false negatives (compounds that are more potent than E2 but were predicted to be less potent) and well-predicted compounds (Table 1). Even though the experimental approach by Badeau et al. [32] was not based on the TBARS method but assessed the antioxidant effect on copper-induced oxidation of lipoproteins through the continuous monitoring of conjugated diene formation, our QSAR Equations 1 and 4-8 still correctly predicted the relative antioxidant capacities of A-ring substituted estrogens. Therefore, the strong influence of $\log$, as well as the contribution of $\mathrm{BDE}, \mathrm{SI}_{\kappa 1}$ and HOMO was certainly confirmed in a general context of LPO inhibition by these E2-related compounds. 
Table 1. QSAR model validation using data reported by Badeau et al. [32]).

\begin{tabular}{cccc}
\hline Equation Number & False Positives $^{\mathbf{a}}$ & False Negatives $^{\mathbf{b}}$ & Correctly Predicted $^{\mathbf{c}}$ \\
\hline 1 & 6 & 1 & 21 \\
2 & 19 & 1 & 8 \\
3 & 17 & 1 & 10 \\
4 & 2 & 3 & 23 \\
5 & 5 & 1 & 22 \\
6 & 6 & 2 & 20 \\
7 & 1 & 7 & 20 \\
8 & 7 & 3 & 18 \\
\hline
\end{tabular}

${ }^{\mathrm{a}}$ False positives are compounds that are less potent than E2 but were predicted to be more potent; ${ }^{\mathrm{b}}$ False negatives are those compounds that are more potent than E2 but were predicted to be less potent; and ${ }^{\mathrm{c}}$ Correctly-predicted are those compounds that were predicted correctly as more or less potent than E2 [32]. Leaving out Equations 2 and 3 that were definitely not validated by this strategy, the overall rate of correct predictions was $74 \%$, while false positives and false negatives were $16 \%$ and $10 \%$, respectively.

\section{Experimental Section}

\subsection{Materials and Methods}

Triton X-100, EDTA, bovin serum albumin (BSA), copper (II) sulfate, bicinchoninic acid (BCA), iron (III) chloride, trichloroacetic acid, hydrochloric acid and thiobarbituric acid (TBA) were purchased from Sigma-Aldrich (St. Louis, MO, USA). All other chemicals and solvents were purchased from Fisher Scientific Company (Pittsburgh, PA, USA). Brains were obtained from 2 to 6 ovariectomized (OVX) Sprague-Dawley rats (Charles River Laboratories, Wilmington, MA, USA), three weeks after ovariectomy to ensure that endogenous E2 and E1 would be negligible in the animals and, therefore, would not be interfering factors in the in vitro studies. All animal procedures were approved by the Animal Care and Use Committee of University of North Texas Health Science Center.

The training data set used to construct the models for the QSAR study was composed of seventy compounds. These compounds were commercially available (Steraloids, Newport, RI or Sigma-Aldrich, St. Louis, MO, USA), or their availability has been specified earlier [25]. They are listed in Table S1, along with their experimentally determined antioxidant potencies against iron(III)-induced LPO in OVX rat brain homogenate expressed as $\mathrm{IC}_{50}$ values (in molar concentration, $\mathrm{M})$ relying on the TBARS method [25,37]. Protein concentrations were determined through bicinchoninic acid assay [51,52]. Briefly, 20\% $w / v$ rat brain homogenate [53] (prepared in aqueous TritonX buffer: TritonX-100, $1 \% v / v$; EDTA $1 \mathrm{mM}$; $\mathrm{NaCl}, 0.9 \% w / v$ ) was diluted into phosphate-buffered saline, $\mathrm{pH} 7.4$, to afford $1 \mathrm{mg} / \mathrm{mL}$ protein concentration. After addition of the test compound from ethanolic stock solution, the mixture was held at room temperature for $30 \mathrm{~min}$. $\mathrm{FeCl}_{3}$ was then added from aqueous stock solution to reach $300 \mu \mathrm{M}$ in its concentration, and the sample was incubated at $37{ }^{\circ} \mathrm{C}$ for $15 \mathrm{~min}$. For the measurement of MDA formation, $150 \mu \mathrm{L}$ of $12.5 \%(v / v)$ trichloroacetic acid in $0.8 \mathrm{~N} \mathrm{HCl}$ and $300 \mu \mathrm{L}$ TBA $(1 \%, w / v)$ solutions were added and incubated for an additional hour at $37^{\circ} \mathrm{C}$. Then, the sample was centrifuged at 12,000 rpm for $2 \mathrm{~min}$. The relative fluorescent units (RFU) of the supernatant was determined at an excitation and emission wavelengths 
of 530 and $590 \mathrm{~nm}$ respectively in a fluorescence FL600 microplate reader (Biotek, Winooski, VT, USA). The percent of inhibition of LPO from the TBAR assay was calculated as follows:

$$
\% \text { inhibition }=\left(1-A / A_{0}\right) \times 100
$$

where $A$ is the absorbance in the presence of the antioxidant compound at various concentrations and $A_{0}$ is the absorbance of the control reaction. Each compound was tested in three independent experiments with five to six different levels of inhibitor concentration in each experiment. Sigmoidal dose-response relationships were presumed. Prism (version 3.0; GraphPad Software, La Jolla, CA, USA, 2005) was used to calculate the $\mathrm{IC}_{50}$ values of the compounds.

\subsection{QSAR}

Computations were performed by using CAChe software (version 6.1.1; Fujitsu, Beaverton, OR, USA, 2006). Structures were preoptimized using augmented MM3 parameters followed by full geometry optimization (RMS gradient $<0.1 \mathrm{kcal} /(\AA \cdot \mathrm{mol})$ ) and energy minimization by using the PM5 semi-empirical method with open-shell wave functions (unrestricted Hartree-Fock (UHF) approach).

QSAR equations were obtained through the Project Leader molecular spreadsheet linked to CAChe. In addition to "built-in" structural, spatial, electronic, quantum-chemical and thermodynamic descriptors available through this module, BDE of the phenolic $\mathrm{O}-\mathrm{H}$ bond [40] was also included for each compound. The latter descriptor was calculated as

$$
\mathrm{BDE}=\mathrm{H}_{\mathrm{r}}+\mathrm{H}_{\mathrm{H}}-\mathrm{H}_{\mathrm{p}}
$$

where $\mathrm{H}_{\mathrm{p}}$ and $\mathrm{H}_{\mathrm{r}}$ were the calculated enthalpies of formation for the parent phenolic molecule and for the phenoxyl radical, respectively, while an experimental value $(52.08 \mathrm{kcal} / \mathrm{mol})$ was used as the enthalpy of formation for the hydrogen atom $\left(\mathrm{H}_{\mathrm{H}}\right)$. The stepwise regression algorithm of the Project Leader module was applied to select appropriate descriptors for building the QSAR models. ANOVA was performed using the Minitab software (version 14; Minitab Inc., State College, PA, USA, 2005) to obtain the $F$-values for the regressions.

\section{Conclusions}

The main finding of our QSAR study reported here is that lipophilicity was the property that influenced most profoundly the potency of estrogen derivatives and related polycyclic phenols to inhibit iron(III)-induced LPO in rat brain homogenate. The contribution of BDE of the phenolic O-H group, a shape index $\left(\mathrm{SI}_{\kappa 1}\right)$ and HOMO was also confirmed by our theoretical approach. Several QSAR Equations 1 and 4-8 were validated as potentially useful exploratory tools for identifying or designing novel phenolic antioxidants incorporating the structural backbone of E2 to inhibit LPO and, thus, to assist the discovery of potential therapeutic interventions that could alleviate oxidative stress-induced neurodegeneration in the brain.

\section{Acknowledgments}

This work was supported by the National Institute of Health (grant numbers NS044765 and AG031535 to L.P.) and the Robert A. Welch Foundation (endowment number BK-0031 to L.P.). The 
authors acknowledge the permission of J.W. Simpkins for the use of his laboratory and also for providing technical support to N.M.R.-P. who performed the TBARS assays that enabled the inclusion of several estrogen derivatives with previously not reported antioxidant potencies into our QSAR study. We thank Alexander Oliferenko for the critical reading of and valuable comments on the manuscript.

\section{Conflict of Interest}

The authors declare no conflict of interest.

\section{References}

1. Mariani, E.; Polidori, M.C.; Cherubini, A.; Mecocci, P. Oxidative stress in brain aging, neurodegenerative and vascular diseases: An overview. J. Chromatogr. B 2005, 827, 65-75.

2. Niki, E. Lipid peroxidation products as oxidative stress biomarkers. Biofactors 2008, 34, 171-180.

3. Wagner, B.A.; Buettner, G.R.; Burns, C.P. Free radical-mediated lipid peroxidation in cells: Oxidizability is a function of cell lipid bis-allylic hydrogen content. Biochemistry 1995, 33, 4449-4453.

4. Butterfield, D.A.; Lange, M.L.B.; Sultana, R. Involvements of the lipid peroxidation product, $\mathrm{HNE}$, in the pathogenesis and progression of Alzheimer's disease. Biochim. Biophys. Acta 2010 , 1801, 924-929.

5. Mancuso, C.; Barone, E.; Guido, P.; Miceli, F.; Di Domenico, F.; Perluigi, M.; Santangelo, R.; Preziosi, P. Inhibition of lipid peroxidation and protein oxidation by endogenous and exogenous antioxidants in rat brain microsomes in vitro. Neurosci. Lett. 2012, 19, 101-105.

6. Mooradian, A.D. Antioxidant properties of steroids. J. Steroid Biochem. Mol. Biol. 1993, 45, 509-511.

7. Prokai-Tatrai, K.; Prokai, L.; Jung, M.E. Phenolic compounds protect cultured hippocampal neurons against ethanol-withdrawal induced oxidative stress. Int. J. Mol. Sci. 2009, 10, 1773-1787.

8. Siriphorn, A.; Chompoopong, S.; Floyd, C.L. 17 $\beta$-estradiol protects Schwann cells against $\mathrm{H}_{2} \mathrm{O}_{2}$-induced cytotoxicity and increases transplanted Schwann cell survival in a cervical hemicontusion spinal cord injury model. J. Neurochem. 2010, 115, 864-872.

9. Wilson, M.E.; Dubal, D.B.; Wise, P.M. Estradiol protects against injury-induced cell death in cortical explant cultures: A role for estrogen receptors. Brain Res. 2000, 873, 235-242.

10. Prokai, L.; Prokai-Tatrai, K.; Perjesi, P.; Zharikova, A.D.; Perez, E.J.; Liu, R.; Simpkins, J.W. Quinol-based cyclic antioxidant mechanism in estrogen neuroprotection. Proc. Nat. Acad. Sci. USA 2003, 96, 8867-8872.

11. Gerstner, B.; Lee, J.; DeSilva, T.M.; Jensen, F.E.; Volpe, J.J.; Rosenberg, P.A. 17beta-estradiol protects against hypoxic/ischemic white matter damage in the neonatal rat brain. J. Neurosci. Res. 2009, 87, 2078-2086.

12. Kii, N.; Adachi, N.; Liu, K.; Arai, T. Acute effects of 17 beta-estradiol on oxidative stress in ischemic rat striatum. J. Neurosurg. Anesthesiol. 2005, 17, 27-32. 
13. Strom, J.O.; Theodorsson, A.; Theodorsson, E. Dose-related neuroprotective versus neurodamaging effects of estrogens in rat cerebral ischemia: A systematic analysis. J. Cereb. Blood Flow Metabol. 2009, 29, 1359-1372.

14. Prokai, L.; Oon, S.M.; Prokai-Tatrai, K.; Abboud, K.A.; Simpkins, J.W. Synthesis and biological evaluation of 17beta-alkoxyestra-1,3,5(10)-trienes as potential neuroprotectants against oxidative stress. J. Med. Chem. 2001, 4, 110-114.

15. Prokai, L.; Simpkins, J.W. Structure-nongenomic neuroprotection relationship of estrogens and estrogen-derived compounds. J. Pharmacol. Ther. 2007, 114, 1-12.

16. Moosmann, B.; Behl, C. The antioxidant neuroprotective effects of estrogens and phenolic compounds are independent from their estrogenic properties. Proc. Natl. Acad. Sci. USA 1999, 96, 8867-8872.

17. Prokai-Tatrai, K.; Perjesi, P.; Rivera-Portalatin, N.M.; Prokai, L. Mechanistic investigations on the antioxidant action of a neuroprotective estrogen derivative. Steroids 2009, 73, 280-288.

18. Hum, P.D.; Macrae, M. Estrogen as a neuroprotectant in stroke. J. Cereb. Blood Flow Metabol 2000, 2, 631-652.

19. Römer, W.; Oettel, M.; Menzenbach, B.; Droescher, P.; Schwarz, S. Novel estrogens and their radical scavenging effects, iron-chelating, and total antioxidative activities: 17-alpha-substituted analogs of delta 9(11)-dehydro-17 beta-estradiol. Steroids 1997, 62, 688-694.

20. Yune, T.E.; Kim, S.J.; Lee, S.M.; Lee, Y.K.; Oh, Y.J.; Kim, Y.C.; Markelonis, G.J.; Oh, T.H. Systemic administration of $17 \mathrm{~b}$-estradiol reduces apoptotic cell death and improves functional recovery following traumatic spinal cord injury in rats. J. Neurotrauma 2004, 21, 293-306.

21. Wu, B.; Kulkarni, K.; Basu, S.; Zhang, S.; Hu, M. First-pass metabolism via UDP-glucuronosyltransferase: A barrier to oral bioavailability of phenolics. J. Pharm. Sci. 2011, 100, 3655-3681.

22. DePaolo, L.; Negro-Vilar, A. Estrogenic feminization of the LH response to orchidectomy in the rat: Evidence for a hypothalamic site of action. Neuroendocrinol 1982, 34, 104-111.

23. Hsueh, A.J.W.; Peck, E.J.; Clark, J.H. Progesterone antagonism of the oestrogen receptor and oestrogen-induced uterine growth. Nature 1975, 254, 337-339.

24. Ruiz-Larrea, M.B.; Martin, C.; Martinez, R.; Navarro, R.; Lacort, M.; Miller, N.J. Antioxidant activities of estrogens against aqueous and lipophilic radicals; differences between phenol and catechol estrogens. Chem. Phys. Lipids 2000, 105, 179-188.

25. Perez, E.; Cai, Z.Y.; Covey, D.F.; Simpkins, J.F. Neuroprotective effects of estratriene analogs: Structure-activity relationships and molecular optimization. Drug Dev. Res. 2005, 66, 78-92.

26. Cummins, C.H. Synthesis and radiochemistry of 2,4-disubstituted 17-iodovinylestradiols. Steroids 1994, 59, 590-596.

27. Miller, C.P.; Jirkovsky, I.; Hayhurst, D.A.; Adelmant, S.J. In vitro antioxidant effects of estrogens with a hindered $3-\mathrm{OH}$ function on the copper-induced oxidation of low density lipoprotein. Steroids 1996, 61, 305-308.

28. Tong, W.; Perkins, R.; Strelitz, R.; Collantes, E.R.; Keenan, S.; Welsh, W.J.; Branham, W.S.; Sheehan, D.M. Quantitative structure-activity relationships (QSARs) for estrogen binding to the estrogen receptor: predictions across species. Environ. Health Perspect. 1997, 105, 1116-1124. 
29. Hu, J.-Y.; Aizawa, T. Quantitative structure-activity relationships for estrogen receptor binding affinity of phenolic chemicals. Water Res. 2003, 37, 1213-1222.

30. Hong, H.; Tong, W.; Fang, H.; Shi, L.; Xie, Q.; Wu, J.; Perkins, R.; Walker, J.D.; Branham, W.; Sheehan, D.M. Prediction of estrogen receptor binding for 58,000 chemicals using an integrated system of a tree-based model with structural alerts. Environ. Health Perspect. 2002, 110, 29-36.

31. Behl, C.; Skutella, T.; Lezoualc'h, F.; Post, A.; Widmann, M.; Newton, C.J.; Holsboer, F. Neuroprotection against oxidative stress by estrogens: Structure-activity relationship. Mol. Pharmacol. 1997, 51, 535-541.

32. Badeau, M.; Adlercreutz, H.; Kaihocaara, P.; Tikkanen, M.J. Estrogen A-ring structure and antioxidative effect on lipoproteins, J. Steroid Biochem. Mol. Biol. 2005, 96, 271-278.

33. Callaway, J.K.; Beart, P.M.; Jarrott, B. A reliable procedure for comparison of antioxidants in rat brain homogenates. J. Pharmacol. Toxicol. Meth. 1998, 39, 155-162.

34. Esterbauer, H.; Cheeseman, K.H. Determination of aldehydic lipid peroxidation products: Malondialdehyde and 4-hydroxynonenal. Methods Enzymol. 1990, 186, 407-421.

35. Mihaljevic, B.A.; Katusin-Razem, B.; Razem, D. The reevaluation of the ferric thiocyanate assay for lipid hydroperoxides with special considerations of the mechanistic aspects of the response. Free Radic. Biol. Med. 1996, 21, 53-63.

36. Janero, D.R. Malondialdehyde and thiobarbituric acid-reactivity as diagnostic indices of lipid peroxidation and peroxidative tissue injury. Free Radic. Biol. Med. 1990, 9, 515-540.

37. Rivera-Portalatin, N.M. Mechanism of the Antioxidant Action of Estrogens. Ph.D. Thesis, University of Florida, Gainesville, FL, USA, 2006.

38. Perez, E.J. Estratriene Neuroprotection through Antioxidant, Non-Estrogen Receptor Mediated Mechanisms. Ph.D. Thesis, University of Florida, Gainesville, FL, USA, 2004, p. 102.

39. Mitra, I.; Saha, A.; Roy, K. In silico development, validation and comparison of predictive QSAR models for lipid peroxidation inhibitory activity of cinnamic acid and caffeic acid derivatives using multiple chemometric and cheminformatics tools. J. Mol. Model. 2012, 18, 3951-3967.

40. Cheng, Z.Y.; Ren, J.; Li, Y.Z.; Chang, W.B.; Chen, Z.D. Establishment of a quantitative structure-activity relationship model for evaluating and predicting the protective potentials of phenolic antioxidants on lipid peroxidation. J. Pharm. Sci. 2003, 92, 475-484.

41. Bakalbassis, E.G.; Chatzopoulou, A.; Malissas, V.S.; Tsimidou, M.; Vafiadis, A. Ab initio and density functional theory studies for the explanation of the antioxidant activity of certain phenolic acids. Lipids 2001, 36, 181-190.

42. Mukai, K.; Uemoto, Y.; Fukuhara, M.; Nagaoka, S.; Ishizu, K. ENDOR study of the cation radicals of vitamin-E derivatives-relation between antioxidant activity and molecular-structure. Bull. Chem. Soc. Jpn. 1992, 65, 2016-2020.

43. Bordwell, F.G.; Zhang, X.-M.; Satish, A.V.; Cheng J.-P. Assessment of the importance of changes in ground-state energies on the bond dissociation enthalpies of the $\mathrm{O}-\mathrm{H}$ bonds in phenols and the S-H bonds in thiophenols. J. Am. Chem. Soc. 1994, 116, 6605-6610.

44. Prokai, L.; Prokai-Tatrai, K.; Perjesi, P.; Simpkins, J.W. Mechanistic insights into the direct antioxidant effects of estrogens. Drug Dev. Res. 2006, 66, 118-125. 
45. Pedulli, G.F.; Lucarini, A.; Pedrielli, P. Bond Dissociation Energies of Phenolic and Amine Antioxidants. In Free Radicals in Biology and Environment; Minisci, F., Ed.; Kluwer Academic Publishers: Dordrecht, The Netherland, 1997; pp. 169-180.

46. Kier, L.B. Indexes of molecular shape from chemical graphs. Med. Res. Rev. 1987, 7, 417-440.

47. Katritzky, A.R.; Gordeeva, E.V. Traditional topological indices vs. electronic, geometric, combined molecular descriptors in QSAR/QSPR research, J. Chem. Inf. Comput. Sci. 1993, 33, 835-857.

48. Klamt, A.; Schüürmann, G. COSMO: A new approach to dielectric screening in solvents with explicit expressions for the screening energy and its gradient. J. Chem. Soc. Perkin Trans. 1993, 2, 799-805.

49. Lorand, T.; Kocsis, B.; Sohar, P.; Nagy, G.; Jozsef, P.; Kispal, G.; Laszlo, R. Prokai, L. Synthesis and antibacterial effect of fused Mannich ketones. Eur. J. Med. Chem. 2002, 37, 803-812.

50. Kocsis, B.; Kustos, I.; Kilar, F.; Nyul, A.; Jakus, P.B.; Kerekes, S.; Villarreal, V.; Prokai, L.; Lorand, T. Antifungal unsaturated cyclic Mannich ketones and aminoalcohols: Study of mechanism of action. Eur. J. Med. Chem. 2009, 44, 1823-1829.

51. Smith, P.K.; Krohn, R.I.; Hermanson, G.T.; Mallia, A.K.; Gartner, F.H.; Provenzano, M.D.; Fujimoto, E.K.; Goeke, N.M.; Olson, B.J.; Klenk, D.C. Measurement of protein using bicinchoninic acid. Anal. Biochem. 1985, 150, 76-85.

52. Walker, J.M. The Bicinchoninic Acid (BCA) Assay for Protein Quantitation. In The Protein Protocols Handbook, 3rd ed.; Walker, J.M., Ed.; Humana Press: New York, NY. USA, 2009; pp. 11-15.

53. Nguyen, V.; Zharikova, A.D.; Prokai-Tatrai, K.; Prokai, L. [Glu ${ }^{2}$ TRH dose-dependently attenuates TRH-evoked analeptic effect in the mouse brain. Brain Res. Bull. 2010, 82, 83-86.

(C) 2013 by the authors; licensee MDPI, Basel, Switzerland. This article is an open access article distributed under the terms and conditions of the Creative Commons Attribution license (http://creativecommons.org/licenses/by/3.0/). 